the cortisone one week group. Intracytoplasmic fine granules were enlarged, but their number decreased remarkably in comparison with the second group. In proportion to the frequency of cortisone administration, the number of extrahepatocellular phosphatide granules increased. And in the liver of alloxan rats, such granules decreased but their distribution in the lobule extended. It is considered that these granules indicated phosphatides partially in Kupffer cells and their number increased in proportion to the accelerated transportation of fat into the liver. It was assumed that the intracytoplasmic fine phosphatide granules enlarged in size and decreased in number in the all diabetic groups, and these granules originated from phosphatides, which are in close contract with swelled mitochondria in liver cells of diabetic animals. The cause of the increase of extracellular phosphatides and the decrease of intrahepatocellular phosphatides observed in the diabetic liver demands further study.

\title{
Histochemical Studies on Lipid Metabolism in Liver of Rats with Experimental Diabetes
}

(2) Effects of Oral Administration of Fats.

Kazutoshi Watanabe and Hajime Mori

2nd Department of Internal Medicine, Gifu University School of Medicine, Gifu

Experimental diabetes in male Wistar rats were induced by the administration of hydrocortisone or alloxan as the previous report. Soybean oil or cod liver oil were administered to diabetic animals in the emulsified form (3m./ $100 \mathrm{gm}$. body weight) through the gastric tube after seventeen hours fasting. In three, six and eight hours after the administration of the oil the animals were sacrificed and their livers were resected and the sections were determined by Sudan Black B (SBB) fat staining. Fresh unfixed sections from another piece of the liver were examined histoenzymologically about the activities of $\beta$-hydroxy butyric acid dehydrogenase $(\beta \mathrm{BDH})$, succinic dehydrogenase $(\mathrm{SDH})$ and adeosine triphosphatase (ATPase). By the SBB staining it was revealed that lipid granules in the liver of normal and diabetic rats increased once in three to six hours after the administration of soybean oil, but after eight hours more or less decreased. By the SBB staining the retardation of emerging and disappearance of liver lipids was found in the diabetic animals especially in the alloxan treated group. In eight hours after the administration of soybean oil, remarkable elevation of activities of $\beta \mathrm{BDH}$ was exhibited especially in the midzone of lobule, corresponding to the localization of the eminent decrease of 
the SBB lipid granules. SDH activities in the liver of the diabetic rats after receiving cod liver oil elevated highly more than the soybean oil received animals. Both in the normal and diabetic groups eight hours after receiving oil, the highest activities of SDH were found to be ditsributed in the part of liver lobule where the highest density of granules by SBB staining was found. Through the observation intervals, the diabetic groups showed evidently higher activities of ATPase in the cytoplasm of the liver cells than the normal rats. Although the activities of $\beta \mathrm{BDH}$ in the liver cells of diabetic rats as well as the normal rats elevated in eight hours after the administration of oil, the degree of the elevation in the cortisone diabetic rats were lower than in normal rats, but the lowest elevation was found in the alloxan rats. The both diabetic groups, eight hours after receiving cod liver oil, showed more remarkably increased activities of ATPase in the liver than in the soybean oil received rats These findings may be explained by the mechanism that the elevations of the ATPase and SDH activities were caused partially by enhanced fatty acid oxidation and partially by the increase of uncoupling of oxidative phosphorylation, and the mechanism that the elevations of the $\beta \mathrm{BDH}$ activities depends on enhanced fatty acid oxidation coupled with synthesis of acetyl CoA. From the above findings it is considered that the assimilation of administered fat in the liver was retarded in the diabetic animals, and the retardation of the assimilation of cod liver oil was more remarkable than that of soybean oil.

\title{
Histochemical Studies of the Brain: Metachromatic Leucodystrophy
}

\author{
Fumitada Hazama, Hideyuki Haebara and Toshio Takeda \\ Department of Pathology, Kyoto University School of Medicine, Kyoto \\ (Director: Professor Kozo OKAMOTO)
}

Metachromatic leucodystrophy is a familial and progressive degenerative disease characterized by the deposition of lipids which shows metachromasia in the cerebral white matter. Biochemically the presence of abnormal increase of sulfatide ${ }^{1,3)}$ which is a sulfuric ester of cerebroside is known in the brain with this disease. Recently, biopsies have been performed in order to differentiate the diseases similar to the cerebral palsy. For this purpose the importance of histochemical differentiation method of lipids has been realized. Although, histochemical studies on the deposited substances in metachromatic leucodystrophy have been less advanced.

\section{Materials and Methods}

This work was performed on materials from one case of 2 years and 7 months old girl with typical metachromatic leucodystrophy. Following stain- 